\title{
ON WEAKLY HARMONIC MAPS AND \\ NOETHER HARMONIC MAPS FROM A RIEMANN SURFACE INTO A RIEMANNIAN MANIFOLD
}

\author{
FRÉDÉRIC HÉLEIN \\ G.H.N., E.N.S.T.A., Centre de l'Yvette \\ Chemin de la Hunière, F-91120 Palaiseau, France
}

I want to present some results on the regularity for harmonic maps between a surface of dimension two and a Riemannian manifold.

First of all, we recall what harmonic maps between Riemannian manifolds are. Let $(\mathcal{M}, g)$ and $(\mathcal{N}, g)$ be two Riemannian manifolds of dimension $m$ and $n$ respectively, and assume that $\mathcal{N}$ is compact and is isometrically embedded in some Euclidean space $\mathbb{R}^{k}$ (which is always possible thanks to the Nash-Moser theorem). We introduce the Dirichlet functional on the set of maps between $\mathcal{M}$ and $\mathcal{N}$. To do this we define the energy density of a map $u$ from $\mathcal{M}$ into $\mathcal{N}$ at any point $x$ of $\mathcal{M}$ by

$$
e(u)(x)=\frac{1}{2} h_{i j}[u(x)] g^{\alpha \beta}(x) u_{\alpha}^{i} u_{\beta}^{j}
$$

if we use local coordinates on $\mathcal{M}$ and $\mathcal{N}$. We may alternativately write the energy density by using the Euclidean structure of the space $\mathbb{R}^{k}$ :

$$
e(u)(x)=\frac{1}{2} g^{\alpha \beta}(x)\left\langle u_{\alpha}, u_{\beta}\right\rangle .
$$

Here $u_{\alpha}$ is the partial derivative $\partial u / \partial x^{\alpha}$. Furthermore, we let the Riemannian volume element be

$$
d v_{g}(x)=\sqrt{\operatorname{det} g_{a b}(x)} d x^{1} \ldots d x^{m} .
$$

Then the energy of a map $u$ is

$$
E(u)=\int_{\mathcal{M}} e(u)(x) d v_{g}(x) .
$$

The function space on which this functional is defined is

$$
H^{1}(\mathcal{M}, \mathcal{N})=\left\{u \in H^{1}\left(\mathcal{M}, \mathbb{R}^{k}\right) \mid u \in \mathcal{N} \text { a.e. }\right\} .
$$


Consider some tubular neighbourhood $\mathcal{V}$ of $\mathcal{N}$ in $\mathbb{R}^{k}$ and let $r: \mathcal{V} \rightarrow \mathcal{N}$ be a smooth retraction of $\mathcal{V}$ onto $\mathcal{N}$. Given a map $u$ in $H^{1}(\mathcal{M}, \mathcal{N})$, for any test map $\varphi$ in $C_{\mathrm{c}}^{\infty}\left(\mathcal{M}, \mathbb{R}^{k}\right)$, and for sufficiently small real $\varepsilon, u+\varepsilon \varphi$ takes its values in $\mathcal{V}$. Therefore we may consider $r(u+\varepsilon \varphi)$ which belongs to $H^{1}(\mathcal{M}, \mathcal{N})$. We say that $u$ is weakly harmonic if and only if

$$
\lim _{\varepsilon \rightarrow 0} \frac{E[r(u+\varepsilon \varphi)]-E(u)}{\varepsilon}=0
$$

for any test map $\varphi$. The Euler equation associated with this type of critical points is

$$
\Delta_{\mathcal{M}} u+A(u)(\nabla u, \nabla u)=0,
$$

where $\Delta_{\mathcal{M}}$ is the Laplace operator on $(\mathcal{M}, g)$, and $A$ depends on the second fundamental form of the embedding of $\mathcal{N}$ into $\mathbb{R}^{k}$.

There exists a second type of critical points for $E$ which we will call Noether harmonic maps, because the associated Euler equation may be deduced by using Noether's theorem from the invariance of the energy functional under the group of diffeomorphisms of $\mathcal{M}$. Consider a smooth family $\Phi_{t}$ of diffeomorphisms of $\mathcal{M}$ such that $\Phi_{0}=$ Id. Then $u$ is Noether harmonic if and only if

$$
\lim _{t \rightarrow 0} \frac{E\left(u \circ \Phi_{t}\right)-E(u)}{t}=0
$$

for any $\Phi_{t}$. The associated Euler equation is that the stress-energy tensor $S=$ $e(u) g-u_{*}(h)$ is divergence free [BE], [B] (here $u_{*}$ denotes the pull-back).

We want to deal here with the regularity question for weakly harmonic maps, or for Noether harmonic maps in the case where $\mathcal{M}$ is a two-dimensional surface. Note that weakly harmonic maps are not regular in general since for example the map $x \rightarrow x /|x|$ from the unit ball of $\mathbb{R}^{3}$ into $S^{2}$ is weakly harmonic but not regular at the origin. However, in the case where $\mathcal{M}$ is a surface, regularity results are known in the following situations:

a) $u$ is energy minimizing (Morrey $[\mathrm{M}]$ ),

b) $u$ is weakly harmonic and conformal (Grüter [Grü]),

c) $u$ is stationary, i.e. weakly harmonic and Noether harmonic (Schoen [Sc]),

d) $u$ is weakly harmonic and its image is contained in a geodesically convex ball (Hildebrandt, Kaul and Widman [HKW]),

e) removability of isolated singularities of harmonic maps (Sacks and Uhlenbeck $[\mathrm{SaU}])$.

In the case where $\mathcal{M}$ is a surface, the energy functional is invariant under conformal transformations, and we may therefore always suppose that locally the metric is flat. Indeed, in complex isothermal coordinates, $z=x+i y$ and using the notations $u_{x}=\partial u / \partial x$ and $u_{y}=\partial u / \partial y$ we have

$$
e(u)(z) d v_{g}(z)=\left(\left|u_{x}\right|^{2}+\left|u_{y}\right|^{2}\right) d x d y,
$$


thus equation (2) becomes

$$
\Delta u+A(u)(\nabla u, \nabla u)=0
$$

where $\Delta=\partial^{2} / \partial x^{2}+\partial^{2} / \partial y^{2}$. The Euler equation associated with the Noether harmonic maps is that the quadratic differential form

$$
\omega=\left[\left|u_{x}\right|^{2}+\left|u_{y}\right|^{2}-2 i\left\langle u_{x}, u_{y}\right\rangle\right](d z)^{2}
$$

is holomorphic ( $\omega$ is called the Hopf differential; note that $\omega \equiv 0$ if and only if $u$ is weakly conformal).

The first result I want to present is related to Noether harmonic maps which are homeomorphisms.

Theorem 1 [H1]. Let $u$ be in $H^{1}(\mathcal{M}, \mathcal{N})$ where $\mathcal{M}$ and $\mathcal{N}$ are Riemannian surfaces of the same genus. Assume that

(i) The Hopf differential $\omega$ is holomorphic.

(ii) $u$ is quasi-conformal, i.e. there exists a real $K$ in $(0,1)$ such that

$$
|\partial u / \partial \bar{z}| \leq K|\partial u / \partial z|
$$

(iii) $u$ is a homeomorphism between $\mathcal{M}$ and $\mathcal{N}$.

Then $u$ is a harmonic diffeomorphism.

Remark. a) In contrast with this result note that J. Jost gave in $[\mathrm{J}]$ an example of a Lipschitz map between the two-dimensional torus and the twodimensional sphere which satisfies (i) but which is not smooth.

b) Since $u$ is a harmonic diffeomorphism it follows from $[\mathrm{CH}]$ that $u$ is energy minimizing.

The second result that I want to present is about the special case where $(\mathcal{N}, h)$ is a round sphere, i.e. a sphere $S^{n}$ equipped with the canonical metric. In this case (3) becomes

$$
\Delta u+u|\nabla u|^{2}=0
$$

where $\Delta$ is the usual Laplace operator on $\mathbb{R}^{2}$. Then we have the following:

THEOREM 2. Let $\mathcal{M}$ be a Riemannian surface and let $S^{n}$ be the canonical sphere of dimension $n$. Then any weakly harmonic map u in $H^{1}\left(\mathcal{M}, S^{n}\right)$ is regular inside $\mathcal{M}$.

This theorem was proved in [H2] (see also [H3]). Here the proof is made shorter and simpler thanks to remarks of P.-L. Lions. In higher dimensions we cannot obtain the same results. However, we may ask if a weakly harmonic map defined on an $m$-dimensional manifold $\mathcal{M}^{m}$ into a sphere which belongs to $W^{1, m}$ (the space of maps which belong to $L^{m}$ and whose first derivatives belong to $L^{m}$ ) is regular or not. In [H3] we also prove

Theorem 3. Let $\mathcal{M}^{m}$ be a Riemannian manifold of dimension $m \geq 3$. Then any weakly harmonic map in $W^{1, m}\left(\mathcal{M}^{m}, S^{n}\right)$ is regular inside $\mathcal{M}^{m}$. 
Another extension of Theorem 2 is to replace the sphere by another Riemannian manifold. In [H3] we prove that the two results above are true if one replaces the sphere by some compact Riemannian manifold on which a Lie group acts transitively by isometries. A basic tool for these extensions is Noether's Theorem. Very recently we found in [H4] the way to generalize Theorem 2 to the case of a manifold without symmetry, where Noether's Theorem is not available.

Also, note that in [E] L. C. Evans used our method in Theorem 2 to prove that any map in $H^{1}\left(\Omega^{m}, S^{n}\right)$ which is stationary (i.e. weakly harmonic and Noether harmonic) is regular on $\Omega^{m} / \mathcal{S}$ where $\mathcal{S}$ is a closed subset of $\Omega^{m}$ whose Hausdorff measure of dimension $m-2$ is zero.

We now give a short description of the proofs of Theorems 1 and 2 .

Sketch of the proof of Theorem 1. There are two cases. The first is when $\omega \equiv 0$; then $u$ is conformal and the regularity follows from the result of M. Grüter [Grü]. The second case is when $\omega \neq \equiv 0$. Then since $\omega$ is holomorphic, $\omega^{-1}(\{0\})$ is a finite collection of points $\left\{a_{1}, \ldots, a_{k}\right\}$. By the result on removability of isolated singularities of [SaU] it suffices to show that $u$ is regular everywhere outside $\omega^{-1}(\{0\})$. Let $B_{1}$ be an open ball in $\mathcal{M}^{2} \backslash \omega^{-1}(\{0\})$. We will show that the restriction of $u$ to $B_{1}$ is minimizing among maps from $B_{1}$ into $B_{2} \equiv u\left(B_{1}\right)$, which is enough to prove that $u$ is regular because of Morrey's result [M].

Because of the density results it suffices to show that for any map $f$ of class $C^{1}$ from $B_{1}$ to $B_{2}$ which agrees with $u$ on $\partial B_{1}$ we have

$$
E_{B_{1}}(f) \geq E_{B_{1}}(u)
$$

where $E_{B_{1}}(f)=\int_{B_{1}} e(f)(z) d v_{g}(z)=\int_{B_{1}} \frac{1}{2}|\nabla f|^{2}(z) d x d y$.

We assume for the moment that $u$ is of class $C^{1}$ (we will explain briefly at the end why the computations which follow are valid in our case). Write $f=u \circ \eta$ where $\eta$ goes from $B_{1}$ to $B_{1}$. We use the notations $\eta=\left(\eta^{x}, \eta^{y}\right), \varphi_{\alpha}=\partial \varphi / \partial \alpha$ for $\alpha=x$ or $y$, and $\left\langle u_{\alpha}, u_{\beta}\right\rangle=h\left(u_{\alpha}, u_{\beta}\right)$. Then we have

$$
|\nabla f|^{2}=\sum_{\alpha=x, y}\left(\eta_{\alpha}^{x}, \eta_{\alpha}^{y}\right)\left(\begin{array}{cc}
\left|u_{x}\right|^{2} & \left\langle u_{x}, u_{y}\right\rangle \\
\left\langle u_{x}, u_{y}\right\rangle & \left|u_{y}\right|^{2}
\end{array}\right)\left(\begin{array}{l}
\eta_{\alpha}^{x} \\
\eta_{\alpha}^{y}
\end{array}\right) .
$$

Equivalently,

$$
\begin{aligned}
|\nabla f|^{2}= & \sum_{\alpha=x, y} \frac{\left|u_{x}\right|^{2}+\left|u_{y}\right|^{2}}{2}\left(\eta_{\alpha}^{x}, \eta_{\alpha}^{y}\right)\left(\begin{array}{cc}
1 & 0 \\
0 & 1
\end{array}\right)\left(\begin{array}{c}
\eta_{\alpha}^{x} \\
\eta_{\alpha}^{y}
\end{array}\right) \\
& +\sum_{\alpha=x, y}\left(\eta_{\alpha}^{x}, \eta_{\alpha}^{y}\right)\left(\begin{array}{cc}
\frac{\left|u_{x}\right|^{2}-\left|u_{y}\right|^{2}}{2} & \left\langle u_{x}, u_{y}\right\rangle \\
\left\langle u_{x}, u_{y}\right\rangle & \frac{\left|u_{y}\right|^{2}-\left|u_{x}\right|^{2}}{2}
\end{array}\right)\left(\begin{array}{c}
\eta_{\alpha}^{x} \\
\eta_{\alpha}^{y}
\end{array}\right) .
\end{aligned}
$$

Now let us use (i). Since $\omega \neq 0$ on $B_{1}$ there exists a holomorphic map $g$ from $B_{1}$ into $\mathbb{C}$ such that

$$
g(z)^{2}=\left|u_{x}\right|^{2}-\left|u_{y}\right|^{2}-2 i\left\langle u_{x}, u_{y}\right\rangle
$$


Let $\theta(z)=\operatorname{Re}\left\{\int_{z_{0}}^{z} g(t) d t\right\} . \theta$ is constructed in such a way that $g(z)^{2}=\left(\theta_{x}\right)^{2}-$ $\left(\theta_{y}\right)^{2}-2 i \theta_{x} \theta_{y}$ (formally the same expression as (6) where $u$ is replaced by $\theta$ ). We can also write

$$
\begin{aligned}
|\nabla(\theta \circ \eta)|^{2}= & \sum_{\alpha=x, y} \frac{\left(\theta_{x}\right)^{2}+\left(\theta_{y}\right)^{2}}{2}\left(\eta_{\alpha}^{x}, \eta_{\alpha}^{y}\right)\left(\begin{array}{ll}
1 & 0 \\
0 & 1
\end{array}\right)\left(\begin{array}{l}
\eta_{\alpha}^{x} \\
\eta_{\alpha}^{y}
\end{array}\right) \\
& +\sum_{\alpha=x, y}\left(\eta_{\alpha}^{x}, \eta_{\alpha}^{y}\right)\left(\begin{array}{cc}
\frac{\left(\theta_{x}\right)^{2}-\left(\theta_{y}\right)^{2}}{2} & \theta_{x}, \theta_{y} \\
\theta_{x}, \theta_{y} & \frac{\left(\theta_{y}\right)^{2}-\left(\theta_{x}\right)^{2}}{2}
\end{array}\right)\left(\begin{array}{l}
\eta_{\alpha}^{x} \\
\eta_{\alpha}^{y}
\end{array}\right) .
\end{aligned}
$$

Now if we compute the difference between (5) and (7), we find

$$
|\nabla f|^{2}=|\nabla(\theta \circ \eta)|^{2}+\frac{|\nabla u[\eta(z)]|^{2}-|\nabla \theta[\eta(z)]|^{2}}{2}|\nabla \eta|^{2},
$$

and a straightforward computation shows that $\lambda[\eta(z)] \equiv|\nabla u[\eta(z)]|^{2}-|\nabla \theta[\eta(z)]|^{2}$ is positive. Thus

$$
|\nabla f|^{2} \geq|\nabla(\theta \circ \eta)|^{2}+\lambda[\eta(z)]\left|\begin{array}{ll}
\eta_{x}^{x} & \eta_{y}^{x} \\
\eta_{x}^{y} & \eta_{y}^{y}
\end{array}\right|,
$$

with equality in the case $\eta=$ id, i.e. $f=u$. By integrating by parts over $B_{1}$ we get $E_{B_{1}}(f) \geq E_{B_{1}}(u)$ since $\theta$ is real harmonic.

Now to justify these arguments we must show that the chain rule for differentiation of composed functions may be used. But hypotheses (i) and (ii) lead precisely to the conclusion that $u^{-1}$ is a Lipschitz map, which is enough to apply the chain rule. This terminates the proof of Theorem 1.

Proof of Theorem 2. First recall two results which will be very useful.

LEMma 1 (Noether's Theorem). Let $u$ be a weakly harmonic map in $H^{1}\left(\mathcal{M}^{2}, S^{n}\right)$. Then for any $i, j$ in $\{1, \ldots, n\}$, the following tangent vector field $b^{i j}$ of class $L^{2}$ on $\mathcal{M}^{2}$ is divergence free in the distribution sense:

$$
b^{i j}=u^{i} \operatorname{grad} u^{j}-u^{j} \operatorname{grad} u^{i} .
$$

This fact was already noticed by J. Shatah [Sh], by Y.-M. Chen [Ch] and by J. Keller, J. Rubinstein, P. Sternberg [KRS]. It can be verified by direct computation. However, this is nothing else but application of Noether's Theorem to the case of harmonic maps into a sphere. Here the symmetries which are used are the isometries in $\mathrm{SO}(n+1)$ which act on $S^{n}$.

We will also use the following nice lemma of H. Wente [W] whose proof can be found in the Appendix of $[\mathrm{BC}]$, or which can be deduced from recent results of R. Coifman, P.-L. Lions, Y. Meyer and S. Semmes on Hardy spaces (see [CLMS] or $[\mathrm{E}])$.

Lemma 2. Let $v$ and $w$ be two maps in $H^{1}\left(B^{2}, \mathbb{R}\right)$ where $B^{2}$ is the unit open ball of $\mathbb{R}^{2}$ and let $\varphi$ be the solution of

$$
\Delta \varphi=v_{x} w_{y}-v_{y} w_{x} \quad \text { on } B^{2}, \quad \varphi=0 \quad \text { on } \partial B^{2} .
$$


Then $\varphi$ is in $H^{1}\left(B^{2}, \mathbb{R}\right) \cap C^{0}\left(B^{2}, \mathbb{R}\right)$.

Now let us turn to the proof of Theorem 2. Here since the expected result is local we may work on the unit open ball $B^{2}$ of $\mathbb{R}^{2}$. Let $u$ be a map in $H^{1}\left(B^{2}, S^{n}\right)$ which is weakly harmonic. We observe that since the norm of $u$ is constant we have $\langle u, \operatorname{grad} u\rangle=0$, and thus we can express $\operatorname{grad} u$ in the following way:

$$
\operatorname{grad} u^{i}=\sum_{j=1}^{n+1}\left(u^{j}\right)^{2} \operatorname{grad} u^{i}-u^{j} u^{i} \operatorname{grad} u^{j}=\sum_{j=1}^{n+1} u^{j} b^{j i},
$$

where the $b^{j i}$ were introduced in Lemma 1.

Now we compute the divergence of this expression to obtain

$$
\Delta u^{i}=\sum_{j=1}^{n+1} u^{j} \operatorname{div}\left(b^{j i}\right)+\operatorname{grad} u^{j} \cdot b^{j i}=\sum_{j=1}^{n+1} \operatorname{grad} u^{i} \cdot b^{j i} .
$$

Here we used Lemma 1 to get $\operatorname{div}\left(b^{j i}\right)=0$. Now we will use Lemma 1 a second time to conclude that for any indices $i$ and $j$, there exists a map $\varphi^{j i}$ in $H^{1}\left(B^{2}, \mathbb{R}\right)$ such that

$$
b^{j i}=\operatorname{curl}\left(\varphi^{j i}\right)=\left(\frac{\partial \varphi^{j i}}{\partial y},-\frac{\partial \varphi^{j i}}{\partial x}\right),
$$

and if we insert this last expression in (8) we find

$$
\Delta u^{i}=\sum_{j=1}^{n+1} \frac{\partial u^{j}}{\partial x} \frac{\partial \varphi^{j i}}{\partial y}-\frac{\partial u^{j}}{\partial y} \frac{\partial \varphi^{j i}}{\partial x} .
$$

Hence we can write $u^{i}=v_{1}^{i}+\ldots+v_{n+1}^{i}+\lambda^{i}$ where each $v_{j}^{i}$ is the solution of

$$
\Delta v_{j}^{i}=\frac{\partial u^{j}}{\partial x} \frac{\partial \varphi^{j i}}{\partial y}-\frac{\partial u^{j}}{\partial y} \frac{\partial \varphi^{j i}}{\partial x} \quad \text { on } B^{2}, \quad v_{j}^{i}=0 \quad \text { on } \partial B^{2},
$$

and $\lambda^{i}$ is real harmonic on $B^{2}$. But from Lemma above each $v_{j}^{i}$ is continuous on the closure of $B^{2}$ and $\lambda^{i}$ is obviously smooth inside $B^{2}$, and in conclusion $u$ is continuous inside $B^{2}$. Smoothness of $u$ follows then from standard results in [LU] or from $[\mathrm{HKW}]$.

\section{References}

[B] P. Baird, Harmonic Maps with Symmetry, Harmonic Morphisms and Deformations of Metrics, Res. Notes in Math. 87, Pitman, 1983.

[BE] P. Baird and J. Eells, A conservation law for harmonic maps, in: Geometry Symposium Utrecht 1980, Lecture Notes in Math. 894, Springer, 1981, 1-25.

[BC] H. Brezis and J.-M. Cor on, Multiple solutions of H-systems and Rellich's conjecture, Comm. Pure Appl. Math. 37 (1984), 149-187.

[Ch] Y.-M. Chen, The weak solutions to the evolution problems of harmonic maps, Math. Z. 201 (1989), 69-74. 
[CLMS] R. R. Coifman, P.-L. Lions, Y. Meyer et S. Semmes, Compacité par compensation et espaces de Hardy, C. R. Acad. Sci. Paris Sér. I 309 (1989), 945-949.

$[\mathrm{CH}] \quad$ J.-M. Coron and F. Hélein, Harmonic diffeomorphisms, minimizing harmonic maps and rotational symmetry, Compositio Math. 69 (1989), 175-228.

[E] L. C. Evans, Partial regularity for stationary harmonic maps into spheres, preprint

[Grü] M. Gr üter, Regularity of weak H-surfaces, J. Reine Angew. Math. 329 (1981), 1-15.

[H1] F. Hélein, Homéomorphismes quasi conformes entre surfaces riemanniennes, C. R. Acad. Sci. Paris Sér. I 307 (1988), 591-596.

[H2] -, Régularité des applications faiblement harmoniques entre une surface et une sphère, ibid. 311 (1990), 519-524.

[H3] -, Regularity of weakly harmonic maps from a surface into a manifold with symmetries, Manuscripta Math. 70 (1991), 203-218.

[H4] —, Régularité des applications faiblement harmoniques entre une surface et une variété riemannienne, C. R. Acad. Sci. Paris Sér. I 312 (1991), 591-596.

[HKW] S. Hildebrandt, H. Kaul and K.-O. Widman, An existence theorem for harmonic mappings of Riemannian manifolds, Acta Math. 138 (1977), 1-16.

[J] J. Jost, A note on harmonic maps between surfaces, Ann. Inst. H. Poincaré Anal. Non Linéaire 2 (1985), 397-405.

[KRS] J. Keller, J. Rubinstein and P. Sternberg, Reaction-diffusion processes and evolution to harmonic maps, preprint.

[LU] O. Ladyzhenskaya and N. Ural'tseva, Linear and Quasilinear Elliptic Equations, Academic Press, New York 1968.

[M] C. B. Morrey, The problem of Plateau on a Riemannian manifold, Ann. of Math. 49 (1948), 807-851, see also C. B. Morrey, Multiple Integrals in the Calculus of Variations, Grundlehren Math. Wiss. 130, Springer, Berlin 1966.

[SaU] J. Sacks and K. Uhlenbeck, The existence of minimal immersions of 2-spheres, Ann. of Math. 113 (1981), 1-24.

[Sc] R. Schoen, Analytic aspects of the harmonic maps problem, in: Math. Sci. Res. Inst Publ. 2, Springer, Berlin 1984, 321-358.

[Sh] J. Shatah, Weak solutions and development of singularities of the $\mathrm{SU}(2) \sigma$-model, Comm. Pure Appl. Math. 41 (1988), 459-469.

[W] H. Wente, An existence theorem for surfaces of constant mean curvature, J. Math. Anal. Appl. 26 (1969), 318-344. 